\title{
Gestapo/Sonderauftrag Linz/ Central Collecting Point München/ Österreichische Galerie/Restitution
}

\section{Zur Geschichte der Kunstsammlung von Mathilde und Gottlieb Kraus in Wien}

Eine museale Sammlung wie jene der Österreichischen Galerie ist in ihrer mehr als einhundertjährigen Geschichte einem steten Wandel unterworfen. ${ }^{1}$ Neben Sammlungszuwächsen durch Schenkungen, Ankäufe und Übernahmen aus anderen Museen stehen diverse Deakzessionen von Kunstwerken. So wurden in den 1950er-Jahren Hauptwerke Egon Schieles wie Kardinal und Nonne oder das Porträt Wally an den Sammler Rudolf Leopold im Tausch abgegeben. Nicht nur der unersetzbare Verlust der 1945 in Schloss Immendorf zerstörten Fakultätsbilder Gustav Klimts, sondern auch Abgaben wie der frühen Landschaft Am Attersee bereits 1923 „dezimierten“ den Klimt-Bestand. Aufgrund der alliierten Rückstellungsgesetze wurden nach dem Zweiten Weltkrieg zwölf Kunstwerke u. a. von Emil Jakob Schindler oder Ferdinand Georg Waldmüller, die während der NS-Zeit erworben worden waren, an ihre rechtmäßigen EigentümerInnen restituiert.

Bruno Grimschitz, der damalige Direktor der Österreichischen Galerie, hatte nach dem „Anschluss“ Österreichs an das Deutsche Reich im März 1938 unter aktiver Ausnützung der geänderten Machtverhältnisse eine offensive Erwerbungspolitik durchgeführt; diese ist in direktem Zusammenhang mit der Enteignung und Zerschlagung ,jüdischer“ Kunstsammlungen durch das NS-Regime zu sehen (vgl. Mayer 2005).

Auf der Grundlage des österreichischen Kunstrückgabegesetzes vom Dezember 1998 (BGBl. I Nr. 181/1998 und BGBl. I Nr. 117/2009) und im Sinne einer kritischen Hinterfragung der eigenen Sammlungspolitik werden sämtliche Erwerbungen des Museums ab 1933 systematisch auf ihre Provenienz, d. h. ihre Herkunft, überprüft. Damit soll geklärt werden, ob Kunstwerke während des NS-Regimes

1 Mit der Eröffnung der Modernen Galerie im Mai 1903 wurde der Grundstein für die heutigen Sammlungen der Österreichischen Galerie Belvedere gelegt. Gründungsintention der von den Secessionisten vehement geforderten Errichtung einer öffentlichen Galerie zeitgenössischer Kunst war die Präsentation des österreichischen Kunstschaffens im internationalen Kontext (vgl. Mayer 2018a). 
ihren häufig ,jüdischen“ EigentümerInnen entzogen worden waren. Dank der Ergebnisse der aktuellen Provenienzforschung sind das besondere Ausmaß und die Planmäßigkeit des NS-Kunstraubes in Österreich sowie die unsensible Haltung in der Erwerbungspolitik der Museen auch nach 1945 belegbar. Fragen nach der Provenienz der Kunstwerke und damit nach der Möglichkeit eines vorangegangenen Vermögensentzuges blieben bis 1998 meist ausgeblendet.

Evident ist, dass der österreichische Staat, repräsentiert durch die Musealund Denkmalamtsbürokratie, mögliche Restitutionen von geraubtem Vermögen nicht als Bring-, sondern als Holschuld erachtete. Dies beweist auch der Umgang mit in der NS-Zeit enteigneten oder zwangsweise veräußerten Objekten aus dem Bestand des Sonderauftrag Linz; ${ }^{2}$ in der Nachkriegszeit von der Republik Österreich als Verfallsgut beansprucht, erfolgte 1963 deren Zuweisung an diverse Bundesmuseen (vgl. Mayer 2018b). Nach der Auffindung der Kunstwerke in österreichischen Bergungsorten wurden diese 1945 in den Central Collecting Point (CCP) in München überführt. Ab den späten 1940er-Jahren beanspruchte das Bundesdenkmalamt die Rückführung der Objekte mit der Auflage, deren Restitution an die VorbesitzerInnen abzuwickeln.

Es entbehrt nicht einer gewissen Ironie, dass das Denkmalamt als zuständige Behörde sowohl für die Sicherstellung bzw. Enteignung von Kunstwerken ab 1938 als auch für deren Rückstellung nach 1945 mitverantwortlich war. Entsprechende Aktenbestände, die eine Identifizierung bzw. Zuordnung ermöglicht hätten, waren und sind im Amtsarchiv vorhanden. Dies wird auch am Beispiel eines Gemäldes aus der Sammlung von Mathilde und Gottlieb Kraus zu zeigen sein.

Der „Code of Ethics“ des Internationalen Museumsbundes ICOM nennt als eine der wesentlichen musealen Aufgaben die Erforschung der Provenienz eines Objekts. ${ }^{3}$ „Mit aller gebotenen Sorgfalt“ soll die lückenlose Herkunftsgeschichte überprüft werden. Die umfassenden Quellen- und Literaturrecherchen in Archiven und Bibliotheken werden notwendigerweise ergänzt durch die Autopsie der Originale in den Depots. Treffend schreibt Uwe Schneede, der frühere Direktor der Hamburger Kunsthalle, unter dem Schlagwort „Auf der anderen Seite der Leinwand“:

Es beginnt schon damit, dass Provenienzforscher sich weniger für die Gemälde als für die Rückseiten der Leinwände, mehr für die Bodenplatten als die Hauptansichten der Objekte

2 Unter der Leitung der Kunsthistoriker Hans Posse und Hermann Voss (ab Dezember 1942) sollte der von Adolf Hitler eingesetzte Sonderauftrag Kunstwerke für das in Linz geplante „Führermuseum“ erwerben (vgl. Schwarz 2004).

$3 \mathrm{http} / /$ icom-oesterreich.at/sites/icom-oesterreich.at/files/attachments/ICOM\%20Code\%20 of\%20Ethics_DT.pdf (25.11.2019). 
interessieren. Denn dort finden sie [...] womöglich die entscheidenden Hinweise auf frühere Eigentümer: Aufkleber, Stempel, Siegel, Kennzeichen, Auktionsnummern. Sie wollen findig, mit Spürsinn und Aktenunterstützung entziffert, zum Sprechen gebracht und schlüssig verkettet werden. (Zit. n. Schulz 2014)

Entsprechend dem Kunstrückgabegesetz restituierte das Belvedere seit 1999 insgesamt 58 Kunstwerke aus 34 Sammlungen; darunter befinden sich zehn Gemälde Gustav Klimts, u. a. die beiden Porträts der Adele Bloch-Bauer und eine frühe Schiele-Landschaft. ${ }^{4}$ Für weitere 13 Kunstwerke aus neun Sammlungen und diverse Druckschriften aus der Bibliothek empfahl der zuständige Beirat die Rückgabe; diese verbleiben bis zur Erbenfeststellung im Bestand des Museums.

Der Bogen der restituierten Werke spannt sich von einer gotischen Tafel des Meisters der Veitslegende aus der Sammlung Friedrich Spiegler über barocke Bronzereliefs aus der Rothschild-Kollektion bis zu Hauptwerken der Malerei des 19. Jahrhunderts u. a. von Friedrich Amerling, Anton Romako oder Ferdinand Georg Waldmüller.

Bereits 2002 hatte der Kunstrückgabebeirat des Bundeskanzleramtes eine Rückgabeempfehlung für mehrere Kunstwerke aus der ehemaligen Wiener Sammlung von Mathilde und Gottlieb Kraus ausgesprochen, darunter ein Rudolf von AltAquarell, Pilgramorgelfuß, aus der Albertina. ${ }^{5}$ Ein Seestück Karoly Markós (Abb. 1) und eine Landschaft August von Pettenkofens (Abb. 2) ${ }^{6}$ aus dem Bestand der Österreichischen Galerie wurden 2004 an die RechtsnachfolgerInnen der Familie Kraus ausgefolgt.

Das Museum hatte 1942 das von der Gestapo beschlagnahmte PettenkofenGemälde angekauft; 1963 war Markós Seestück in treuhändige Verwahrung übernommen worden, als eines jener der Republik Österreich verfallenen Kunstwerke, die sich nach Kriegsende im CCP München befanden. Wie auszuführen sein wird, war die Identifizierung der Bilder bzw. deren Zuordnung zur Sammlung Kraus auch möglich durch diverse Aufkleber auf den Bildrückseiten, die damit zur wesentlichen historischen Quelle wurden.

4 https://www.belvedere.at/forschung/provenienzforschung (13.1.2020).

5 Vgl. http://www.provenienzforschung.gv.at/beiratsbeschluesse/Kraus_Gottlieb_2002-06-19. pdf. (25.11.2019).

6 Karoly Markó, Seestück mit Ino und Melikertes; August von Pettenkofen, Zigeunergespann an einer Furt. 


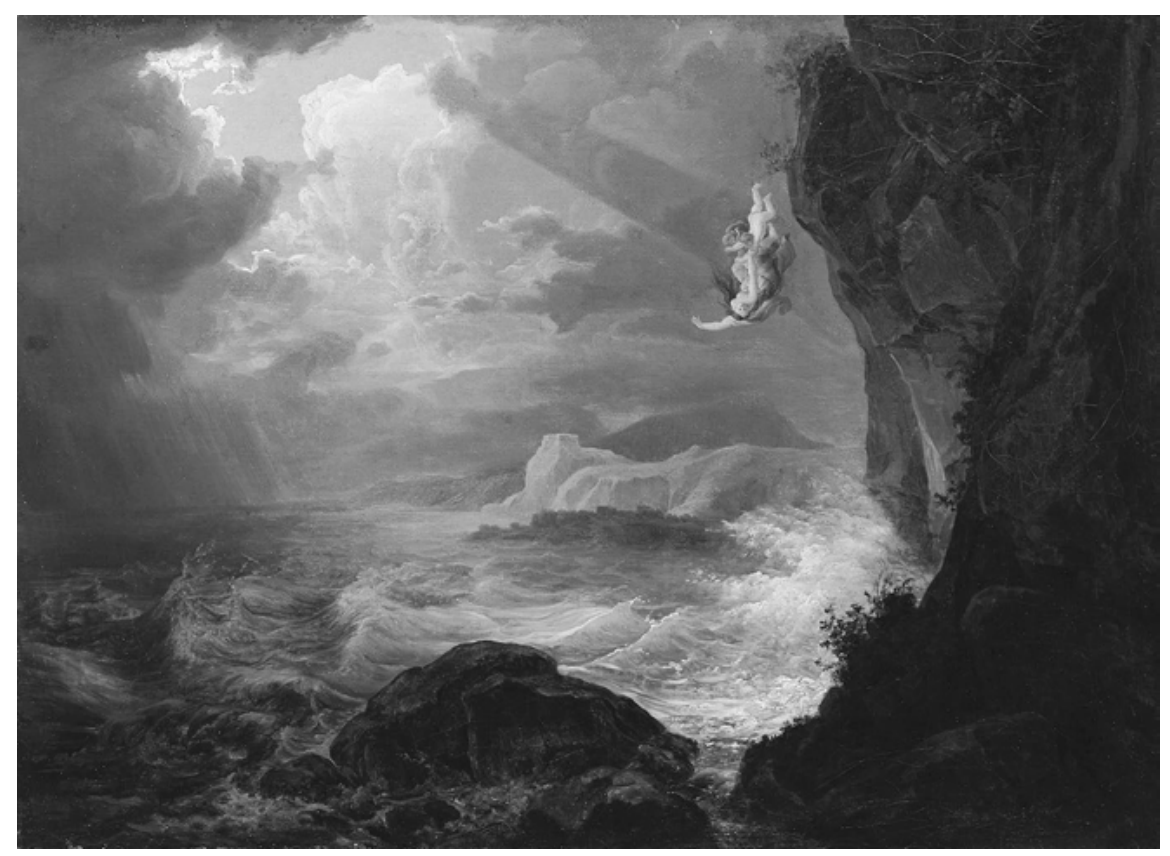

Abb. 1: Karoly Markó: Seestück mit Ino und Melikertes.

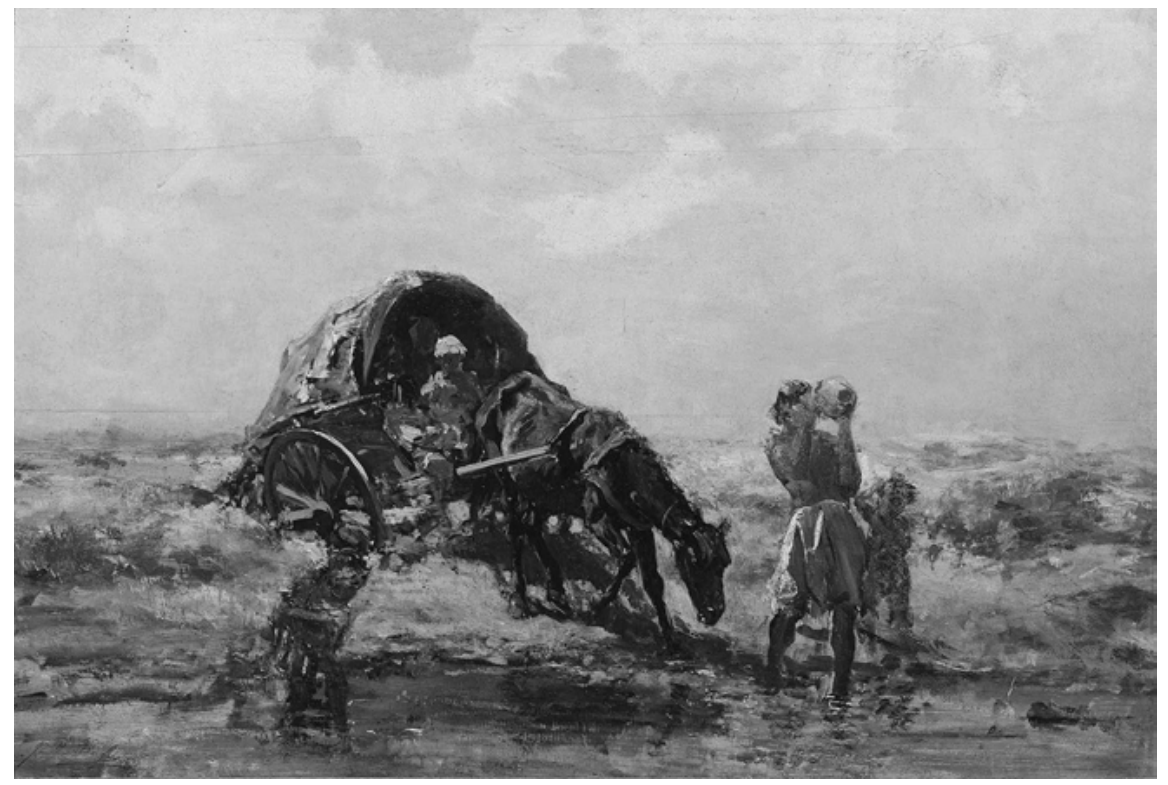

Abb. 2: August von Pettenkofen: Zigeunergespann an einer Furt. 
Gottlieb Bohumil Kraus, geboren 1867 in Kralowitz/Křivsoudov in Böhmen, war tschechoslowakischer Konsul in Wien. Er war verheiratet mit Mathilde Kraus, née Reif, die 1873 in Ungarisch Ostrau zur Welt kam (zur Sammlung Kraus vgl. Lillie 2003, 596-600). Das Ehepaar lebte in einer großzügig ausgestatteten Wohnung in der Wiener Wohllebengasse $16,{ }^{7}$ in unmittelbarer Nähe der barocken Schlossanlage des Belvedere.

Ein Referent des Denkmalamtes hatte im Jänner 1923 die Wohnung der Familie Kraus besichtigt und die Sammlung mit „guten Bildern von Pettenkofen, Gauermann, Waldmüller, J. Emil Schindler, Ribarz, Rudolf Alt, Markó und modernen Malern“ (Archiv BDA, A) ${ }^{8}$ zur öffentlichen Besichtigung gewidmet. Wie häufig auch bei anderen Fällen umfasst der vorliegende Notariatsakt kein detailliertes Sammlungsinventar.

Der „Anschluss“ Österreichs an das nationalsozialistische Deutsche Reich im März 1938 wurde auch für die Familie Kraus zur schrecklichen Zäsur, die Beraubung und Vertreibung zur Folge hatte. Als „Juden“ waren die Familienmitglieder den rassistischen Repressionen des NS-Regimes ausgesetzt. Im Zuge der „Verordnung über die Anmeldung des Vermögens von Juden“ vom 26. April 1938 mussten auch Mathilde und Gottlieb Kraus sogenannte Vermögenserklärungen vorlegen. Ihre Kunstsammlung wurde ohne genauere Beschreibung nur kursorisch mit „Bilder 4.000.- RM“ angeführt (Staatsarchiv, A).

Dem Ehepaar gelang gemeinsam mit der Tochter Marie die Flucht über Prag, Den Haag, Paris und London nach Montreal, Kanada, und weiter in die USA. Das gesamte Vermögen wurde im Juni 1941 mit Verfügung der Gestapo beschlagnahmt; ${ }^{9}$ darunter auch die bei der Spedition Kirchner \& Co. deponierte Kunstsammlung. Der anlässlich der Flucht im Jahr 1938 an die Spedition übergebene Lift war nicht mehr abgefertigt worden. Das beschlagnahmte Übersiedlungsgut der Familie Kraus wurde nach und nach über die Vugesta, die Verwaltungsstelle jüdischen Umzugsgutes der Gestapo, veräußert. Wie den im Österreichischen Staatsarchiv überlieferten Geschäftsbüchern der Vugesta zu entnehmen ist, wurden durch die Verkäufe mehr als 68.000 Reichsmark lukriert. Laut Sophie Lillie einer der größten Einzelerlöse, der je von der Vugesta erzielt wurde (vgl. Lillie 2003, 597).

7 Bekanntheit erlangte die Adresse Wohllebengasse durch Tim Bonyhadys gleichnamiges Buch über die Geschichte der Familie Gallia (vgl. Bonyhady 2013).

$8 \mathrm{Zu}$ den unterschiedlichen Archivmaterialien, aus denen im Folgenden zitiert wird, vgl. die „Archiv-Quellen“ im Anschluss an den Beitrag.

9 „Das gesamte stehende und liegende Vermögen sowie alle Rechte und Ansprüche des Gottlieb Israel Kraus [...] und seiner Ehefrau Mathilde Sara [...] wird aus Gründen der öffentlichen Sicherheit und Ordnung mit dem Ziele der späteren Einziehung zu Gunsten des Deutschen Reiches beschlagnahmt“ (Staatsarchiv, B). 
Unter der Nummer 4423 ist in Band 7 der Vugesta-Journalbücher die Verwertung des Vermögens von Gottlieb Kraus detailliert aufgeschlüsselt (vgl. Staatsarchiv, C). In der angefügten Auflistung der Käufer scheint namentlich der Direktor der Österreichischen Galerie, Bruno Grimschitz, auf. Auch die weiteren angeführten Namen der Erwerber sind Beleg für die Qualität der Kunstsammlung Kraus: genannt seien exemplarisch Museen wie die Graphische Sammlung Albertina und das Landesmuseum Joanneum in Graz, aber auch Hans Posse, Sonderbeauftragter Adolf Hitlers für das „Führermuseum“ in Linz, sowie Heinrich Hoffmann, Hitlers Leibfotograf, der 1943 Karoly Markós Seestück ankaufte.

Im Dezember 1941 übernahm das Institut für Denkmalpflege aus dem Lager der Spedition Kirchner \& Co. zwölf Kunstwerke der Sammlung Kraus zur fotografischen Dokumentation. Auf der im Archiv des Bundesdenkmalamtes verwahrten Übernahmeliste findet sich der Vermerk: „Beschlagnahmungen der Vugesta Wien, I. Bauernmarkt“ (Archiv BDA, B; Abb. 3). Der Pettenkofen scheint als Nr. 7 unter dem Titel Zigeunerwagen am Wasser auf; als Nr. 9 ist die Heroische Seelandschaft mit stürzenden Figuren von Markó angeführt.

Bereits am 7. Jänner 1942 übermittelte Herbert Seiberl, der Leiter des Instituts für Denkmalpflege, Hans Posse für den Sonderauftrag Linz

Fotografien von 12 Bildern, welche mir die Vugesta vorgelegt hat. Es befinden sich darunter einige Gemälde von großartiger Qualität. Vor allem muss auf den Waldmüller, Motiv vom Hallstättersee, hingewiesen werden, dessen Köstlichkeit auf der Fotografie nicht deutlich wird. Ebenso schön ist die Donaulandschaft von Jak. Emil Schindler. Auch die Pettenkofen und R. v. Alt sind sehr schön. Die holländische Landschaft v. J. Emil Schindler ist ebenfalls ein großartiges Werk. Ich bitte um Nachricht, welches von den Bildern für Linz in Betracht käme [...]. (Archiv BDA, C)

Wie dem im Bundesarchiv Koblenz überlieferten Briefwechsel von Hans Posse und dem Beauftragten der Vugesta, Karl Herber, zu entnehmen ist, erfolgte im Februar 1942 tatsächlich die Erwerbung von drei Kunstwerken „,aus der Sammlung Gottlieb Kraus“ für das geplante „Führermuseum“ in Linz. ${ }^{10}$ Hans Posse bestätigte Anfang März den erfolgten Ankauf dreier Bilder von Alt, Schindler und Waldmüller ,von der Vugesta“:

Auf das Aquarell „Der Kanzelfuss in St. Stephan“ von Rud. v. Alt habe ich zu Gunsten der Wiener Stelle, die dieses Bild zu haben wünschte, verzichtet. [...] Die anderen Stücke aus

10 „Laut Mitteilung des Institutes für Denkmalpflege in Wien, Zl. 1325/K 41 wurden für das Kunstmuseum in Linz die nachstehenden 4 Bilder übernommen: 1 . Schindler ,Innlandschaft‘ $\$ 5$. Waldmüller ,Hallstatt‘/11. Rud. v. Alt ,Römischer Triumphbogen`/12. Rud. v. Alt ,Kanzelfuss in St. Stephan““ (Bundesarchiv Koblenz, A). 


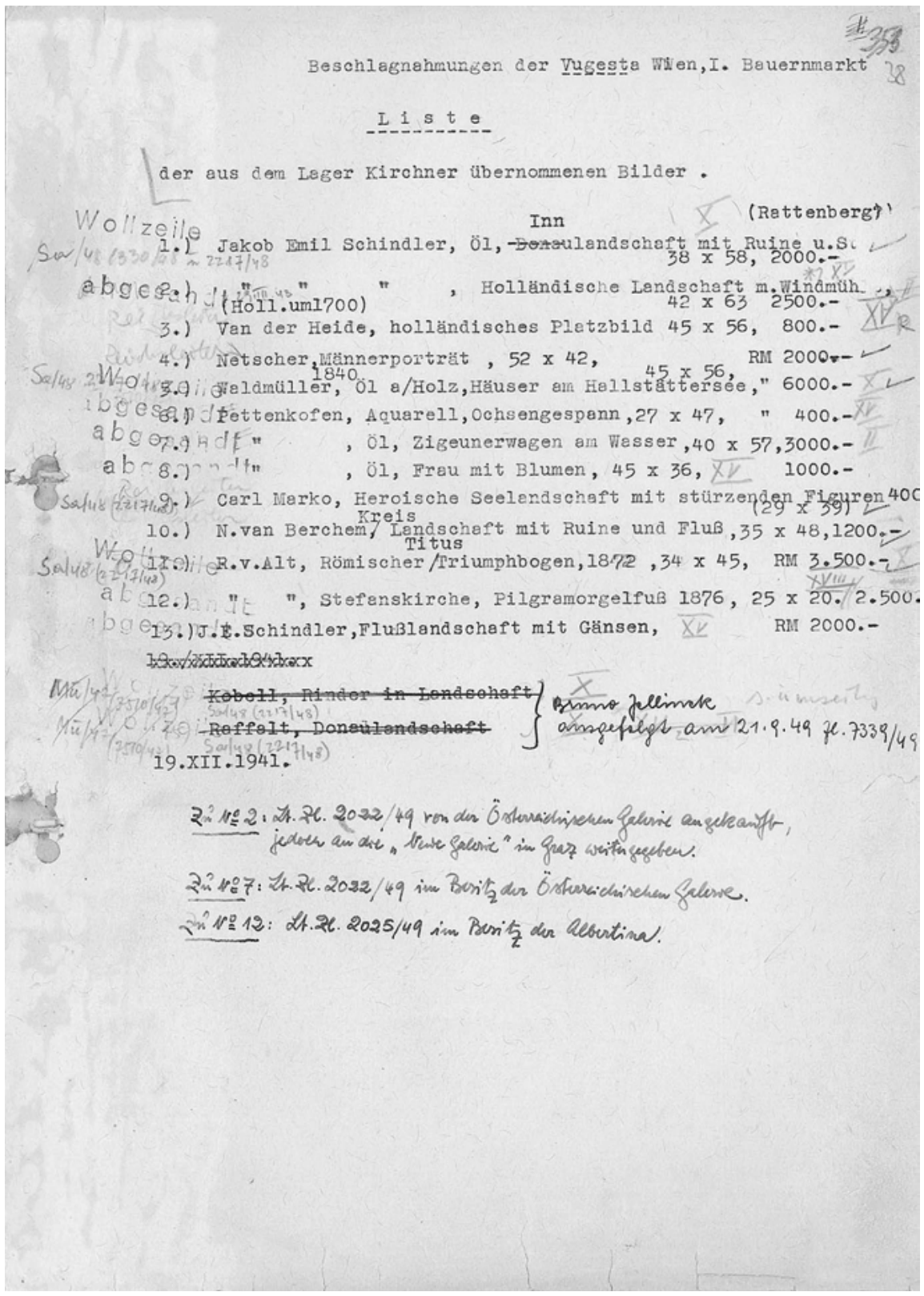

Abb. 3: Beschlagnahmungen der Vugesta, Wien. 
der Masse der Vugesta, [...] stehen zum Kauf für die interessierten Museen frei. (Archiv BDA, D) $)^{11}$

In der Folge erwarb die Österreichische Galerie im April 1942 das Gemälde Zigeunergespann von August Pettenkofen um 3.000 RM und Emil Jakob Schindlers Holländische Landschaft mit Windmühle um 2.500 RM (Archiv Belvedere, A). ${ }^{12}$ Letzteres wurde an das Landesmuseum Joanneum in Graz abgetreten und im September 2004 an die Erben der Familie Kraus restituiert. ${ }^{13}$ Heinrich Hoffmann, Hitlers Leibfotograf, erwarb 1943 Karoly Markós Seestück um 9.800 RM. ${ }^{14}$

Mit dem 16. und 17. US-amerikanischen Transport vom 15. März 1948 wurden die für das „Führermuseum“ erworbenen Werke aus der ehemaligen Sammlung Kraus als „Vugesta-Erwerbungen“ aus dem CCP München nach Salzburg in das Residenzdepot des Bundesdenkmalamtes überstellt; unter den rückgeführten Kunstgegenständen „Unbekannter Eigentümer“ scheint auch Markós Seestück auf (vgl. Archiv BDA, E). Wie erwähnt beanspruchte das Denkmalamt ab den späten 1940er-Jahren die Rückführung der im CCP München deponierten Kunstwerke mit einer österreichischen Provenienz; diese sogenannte äußere Restitution war verbunden mit der Auflage, deren Rückgabe an die rechtmäßigen VorbesitzerInnen abzuwickeln. Ergänzt wird die Auflistung der nach Salzburg transportierten Werke durch die folgende „Anmerkung“:

Auf Bild von Schindler, Schloss Rattenberg: Rückwärts: 590/253 [sic!] Kirchner \& Co [...]

Auf Bild von Waldmüller, Bergsee: 590/245 Kirchner \& Co [...]

Auf Bild von C. Marko, Seestück: Kirchner \& Co. E. Fall Nr. 590/178 (grün) (rot) [...]

Auf Bild von R. Alt, Titusbogen: 3166, Kirchner \& Co. 590/253 [sic!]. (Archiv BDA, E)

Damit erscheint nicht nur auf den drei für die Sammlung Kraus gesicherten Werken von Alt (Titusbogen), Schindler (Rattenberg am Inn) und Waldmüller (Hallstättersee) (vgl. Bundesarchiv, A) ein Klebeetikett der Spedition Kirchner \& Co. - E. Fall, Wien mit der Chiffre „590“ (als Namenskürzel); die Bezeichnung

11 Das im Zitat angesprochene Alt-Aquarell wurde im Juli 1942 von der Albertina um 2.500 RM angekauft.

12 Vgl. besonders den Eintrag in Band 7 der Journalbücher der Vugesta: Nummer 4423, Kraus Gottlieb/IV Wohllebeng. 16/1/5. Angeführt ist der Direktor der Österreichischen Galerie, Bruno Grimschitz, mit den beiden entsprechenden Einzelbeträgen von 3.000 bzw. 2.500 RM: „Dr. Grimschitz, 8.VII.42“ (Staatsarchiv, C).

13 Vgl. https://www.museum-joanneum.at/neue-galerie-graz/ueber-uns/restitution/restituierteobjekte/an-die-erben-nach-gottlieb-und-mathilde-kraus (25.11.2019).

14 Vgl. den Eintrag in Band 7 der Journalbücher der Vugesta (Staatsarchiv, C): „Hofmann [sic!], 17.VI.43, 9.800.-“. 
„590“ findet sich auch auf den rückseitigen Aufklebern an den beiden Werken von Markó und Pettenkofen, ehemals im Bestand der Österreichischen Galerie, und von Schindlers Holländischer Landschaft (590/200), restituiert von der Neuen Galerie in Graz. ${ }^{15}$ Aufgrund der erhaltenen Klebeetiketten mit der identen Chiffre „590“ ist evident, dass es sich bei den auf der Vugesta-Liste vom 19. Dezember 1941 angeführten Kunstwerken um entzogenes Vermögen von Mathilde und Gottlieb Kraus handelt (vgl. Archiv BDA, B). Zu verweisen ist in diesem Kontext auch auf die entsprechenden Eintragungen in den Journalbüchern der Vugesta, wo in Band 7 unter Nummer 4423 die Verwertung des Vermögens von Gottlieb Kraus vermerkt ist. ${ }^{16}$

Aufgrund der mit 17. September 1946 in Kraft getretenen Vermögensentziehungs-Anmeldungsverordnung, die der Erfassung arisierter und anderer entzogener Vermögen dienen sollte, übermittelte der interimistische Leiter der Österreichischen Galerie, Fritz Novotny, an das zuständige Magistratische Bezirksamt im Herbst 1946 eine Aufstellung von 26 Kunstwerken, „die im Lauf der Jahre der nationalsozialistischen Herrschaft in Österreich aus jüdischem Besitz für die Österreichische Galerie erworben wurden“ (Archiv Belvedere, B).

Diese stammen aus acht namentlich genannten Sammlungen - Josef Blauhorn, Viktor Ephrussi, Wilhelm Freund, Gertrud Felsöványi, Irma Götzl, Robert Mendelssohn, Robert Pollak, Leopold Weinstein - bzw. hatten eine unbekannte Provenienz. Darunter waren Ankäufe aus dem Wiener Dorotheum und von der Vugesta bzw. Überweisungen der Reichsstatthalterei. Auch das 1942 erworbene Pettenkofen-Gemälde Zigeunergespann wurde mit dem Vermerk „Unbekannter Eigentümer“ und als Ankauf von der Vugesta angemeldet (vgl. Archiv Belvedere, B). Eine Zuordnung des Gemäldes zur Sammlung Kraus, die aufgrund der vorliegenden Unterlagen im Archiv des Bundesdenkmalamtes leicht möglich gewesen wäre, erfolgte in der unmittelbaren Nachkriegszeit und nur fünf Jahre nach der Beschlagnahmung durch die Gestapo nicht. Auf entsprechende Nachforschungen des Bundesdenkmalamtes über den Verbleib beschlagnahmter Kunstwerke, die von der Vugesta verwertet worden waren, bestätigte die Österreichische Galerie 1949, dass „das Bild von Pettenkofen ,Zigeunerwagen am Wasser“ von der

$15 \mathrm{Vgl}$. https://www.museum-joanneum.at/neue-galerie-graz/ueber-uns/restitution/restituierte-objekte/an-die-erben-nach-gottlieb-und-mathilde-kraus (25.11.2019).

16 Unter den angeführten Käufern scheint nicht nur der Direktor der Österreichischen Galerie, Bruno Grimschitz, auf, sondern auch „Dr. Posse 10.I.42 11.500.-“, „Albertina 8.VII.42 2.500.-“ die Neue Galerie: „Graz 14.IV.42 3.400.-“ und Heinrich Hoffmann, „17.VI.43, 9.800.-“ (siehe die Journalbücher der Vugesta: Nummer 4423, Kraus Gottlieb/IV Wohllebeng. 16/1/5; Staatsarchiv, C). 
Österreichischen Galerie im Jahre 1942 aus den Beständen der Vugesta erworben wurde“ (Archiv Belvedere, C).

Die in den USA im Exil lebende Familie Kraus beauftragte im Jahr 1947 ihren Wiener Rechtsanwalt Otto Scheff, ,alle Schritte zu unternehmen, welche notwendig sein mögen, um unser unbewegliches oder bewegliches Vermögen in Österreich wiederzuerlangen“ und

unsere Ansprüche auf Wiedererstattung oder Schadenersatz für den Verlust von irgendwelchen und allen genannten Grundstücken, Häusern und Einrichtungsstücken aus denselben und die Einrichtung einer zu Wien IV., Oesterreich, Wohllebengasse 16 gelegenen Wohnung vorzubereiten, geltend zu machen und zu vertreten, mit Einschluss von Möbeln und Hausgerät, Orientteppichen, Teppichen anderer Art, einer Sammlung echter, berühmter Gemälde, Kunstgegenständen, antikem Porzellan, Silberwaren, Schmucksachen und aller sonstigen Gegenstände, welche die Einrichtung der genannten Häuser und Wohnung bildeten [...]. (Staatsarchiv, D) ${ }^{17}$

1956 wurde erfolglos ein Antrag auf Rückstellung von drei Bildern aus der Sammlung Kraus an das Joanneum in Graz gerichtet: dabei wurde explizit als entzogenes Vermögen auch August Pettenkofens 1942 von der Österreichischen Galerie erworbenes Gemälde Zigeunerwagen am Wasser angeführt:

Ich erlaube mir, Ihnen nunmehr mitzuteilen, daß sich in vorliegender Sache unter den mir bekanntgegebenen und Ihnen zugewiesenen Bildern nachfolgende im ausschließlichen Besitz und Eigentum meiner Mandanten, der Ehegatten Gottlieb und Mathilde Kraus, zuletzt wh. Wien IV., Wohllebengasse 16 bzw. Wien 19., Grinzingerstraße 36-38 befunden haben:

Jakob Emil Schindler „Bauernhof“

August v. Pettenkofen „Zigeunerwagen am Wasser“

August v. Pettenkofen „Frau mit Blumen“.

Daß diese Bilder ausschließliches Eigentum meiner eingangs erwähnten Klienten sind, geht daraus hervor, daß dieselben durch Herrn akadem. Maler Prof. Ranzoni geschätzt wurden und in dieser Schätzung ausdrücklich angeführt sind. (Steiermärkisches Landesarchiv, A)

Es sollte allerdings bis September 2004 dauern, dass insgesamt drei Gemälde Schindlers und Pettenkofens aus dem Bestand der Grazer Neuen Galerie an die

17 Dem Antrag Otto Scheffs auf Rückstellung einer Liegenschaft in Wien-Grinzing vom 17. September 1947 wurde mit Bescheid der Finanzlandesdirektion für Wien, Niederösterreich und Burgenland vom 8. Mai 1948 auf der Grundlage des Ersten Rückstellungsgesetzes stattgegeben (vgl. Staatsarchiv, D). 
RechtsnachfolgerInnen von Mathilde und Gottlieb Kraus ausgefolgt werden sollten. ${ }^{18}$

Wie bereits ausgeführt, befand sich das von dem Münchener Fotografen Heinrich Hoffmann 1943 erworbene Gemälde von Karoly Markó unter den 1948 von den US-Behörden aus dem CCP München nach Salzburg ins Residenzdepot rückgeführten Kunstgegenständen unbekannter Eigentümer aus Vugesta-Erwerbungen (vgl. Archiv BDA, E). Als eines jener der Republik Österreich verfallenen Kunstwerke wurde Markós Seestück 1963 von der Österreichischen Galerie in treuhändige Verwahrung übernommen (vgl. Archiv Belvedere, D); ${ }^{19} 1965$ erfolgte die definitive inventarische Übernahme (vgl. Archiv Belvedere, E). Auch in diesem Fall unterblieb die Prüfung der Provenienz des Bildes. Ein Blick nicht nur in die Aktenbestände des Denkmalamtes, sondern auch auf die Bildrückseite hätte eine Zuordnung zur Sammlung Kraus ermöglicht.

Offen bleiben muss, warum das in den Akten als „Vugesta-Erwerbung“ geführte Seestück von Karoly Markó als angeblich erbloses Kunstwerk nicht an die sogenannten Sammelstellen ${ }^{20}$ gelangte. In Folge des österreichischen Staatsvertrages von 1955 wurde die individuelle Restitution der Nachkriegszeit in den 1960er-Jahren durch die kollektive Entschädigung über die Sammelstellen abgelöst. Für jene drei aus der Sammlung Kraus stammenden Werke von Rudolf von Alt, Emil Jakob Schindler und Ferdinand Georg Waldmüller, die 1942 über die Vugesta an den Sonderauftrag Linz verkauft wurden, hatte die Sammelstelle A im Juni 1961 einen Rückstellungsantrag gestellt. Bezug nehmend darauf stellte das Bundesministerium für Finanzen fest,

dass die in Rede stehenden Kunstgegenstände für das seinerzeit geplante „Linzer Kunstmuseum“ (auch „Linzer Führermuseum“) bestimmt waren. Dieses Kunstgut stellt, gleichviel ob es sich um persönliches Eigentum Adolf Hitlers oder um Reichseigentum gehandelt haben mag, der Republik Österreich verfallenes Vermögen dar und steht daher in ha. Verwaltung. Verwahrende Stelle ist das Bundesdenkmalamt (BDA), in dessen Salzburger ResidenzDepot sich die Gegenstände befinden. [...] Soweit aus den sehr dürftigen Unterlagen, die

18 Vgl. https://www.museum-joanneum.at/neue-galerie-graz/ueber-uns/restitution/restituierte-objekte/an-die-erben-nach-gottlieb-und-mathilde-kraus (25.11.2019).

19 Rechtsgrundlage für die sogenannten 1963er-Zuweisungen war ein Erlass des Unterrichtsministeriums vom 29. Juni 1963, der „das Bundesdenkmalamt ermächtigt eine Anzahl von Kunstgegenständen, welche seinerzeit für das von Hitler geplante Linzer Kunstmuseum bestimmt waren und in nächster Zeit dem Bundesministerium für Unterricht für die zuständigen Sammlungen des Bundes ressortmäßig übergeben werden, bereits jetzt diesen Sammlungen in treuhändige Verwahrung zu übergeben [...]“ (Archiv Belvedere, D).

20 Zur Tätigkeit der für erblos gebliebene Vermögenswerte zuständigen Sammelstellen siehe Wladika (2018, 85-98). 
vom Bundesdenkmalamt bereits an die Finanzlandesdirektion Salzburg übersendet wurden, hervorgeht, sind dies Objekte, deren Vorbesitzer seinerzeit offenbar Juden waren, bzw. die aus sogenannten VUGESTA-Erwerbungen stammen; aller Voraussicht nach dürfte es sich daher um entzogenes, jedoch nicht beanspruchtes Gut handeln. Die Gegenstände wurden daher im Sinne der Bestimmungen des Auffangorganisationsgesetzes, BGBl. Nr. 73/1957, den Sammelstellen bekanntgegeben. [...] Hingegen wird darauf Bedacht zu nehmen sein, dass im Falle einer Stattgebung der Rückstellungsanträge eine Ausfolgung der Objekte nur nach Begleichung der aus der Rückführung und Lagerung des Kunstgutes entstandenen Spesen [...] erfolgen kann. (Staatsarchiv, E)

Das Bild Donaulandschaft mit Ruinen und Stadt (auch Rattenberg am Inn) von Schindler, eine Waldmüller-Landschaft, der Römische Triumphbogen Rudolf von Alts wurden schließlich 1963 nach einem Rückstellungsvergleich als angeblich „erbloses Vermögen“ an die Sammelstellen ausgefolgt und in der Folge zugunsten von NS-Opfern verwertet. Bereits im September 1961 waren die drei Werke explizit Gegenstand eines Schriftstückes der Treuhandverwaltung von Kulturgut München gewesen. Unter dem Betreff „Rückerstattungssache Kraus, Gottlieb“ wird wie folgt ausgeführt:

[V]on den von Ihnen aufgeführten Bildern wurden die lfd. Nr. 54 Flußlandschaft mit Burg von Schindler, Nr. 85 Triumphtor Aquarell von Rom, 14.12.1872 Sign. unl. (von Rudolf von Alt) sowie Nr. 78 Gehöft am See (von Waldmüller) im Januar 1942 von der VUGESTA an den Sonderauftrag Linz verkauft. Alle drei Werke sind 1948 vom amerikanischen Central Collecting Point München nach Wien als unbekannter Besitz restituiert worden. (Bundesarchiv, B)

Erwähnung finden aber auch weitere Werke aus der Kunstsammlung Kraus, darunter Markós Seestück: „Das Bild lfd. Nr. 86 Engelgruppe von C. Marco dürfte identisch mit dem unter Mü.Nr. 27827 gleichfalls vom Central Collecting Point 1948 restituierten Bild sein, das der ehemalige Reichsbildberichterstatter Heinrich Hoffmann erworben hatte“ (Bundesarchiv, B). Damit kann in Bezug auf die erfolglosen Restitutionsbemühungen der Familie Kraus dem Diktum Sophie Lillies folgend durchaus von einer „zweiten Enteignung“ (Lillie 2008, 220) durch den österreichischen Staat gesprochen werden. Lillie hatte in Bezug auf den sogenannten Mauerbach-Kunstbestand zu Recht auf „das eklatante Versäumnis [hingewiesen], Objekte trotz Wissens um deren Herkunft nicht an die rechtmäßigen EigentümerInnen zu restituieren“ (Lillie 2008, 217). Der „Mauerbach-Bestand“ ist benannt nach dem Aufbewahrungsort in der ehemaligen Kartause Mauerbach in Niederösterreich. Es handelt sich dabei um jene Kunstobjekte, die von den Alliierten im Jahr 1955 mit der Auflage, die rechtmäßigen EigentümerInnen zu suchen und Rückstellungen zu tätigen, an die Republik Österreich übertragen wurden. Der Restbestand wurde 1996 bei der „Mauerbach-Benefizauktion“ durch Christie’s im Wiener MAK-Museum für angewandte Kunst versteigert (vgl. Christie’s Wien 1996). 
Fast exakt 61 Jahre nach der Beschlagnahmeverfügung der Gestapo vom 13. Juni 1941 wurde mit Beschluss des Beirates vom 19. Juni 2002 die Rückgabe des Gemäldes Seestück mit Ino und Melikertes von Karoly Markó und einer Landschaft August Pettenkofens an die RechtsnachfolgerInnen von Mathilde und Gottlieb Kraus empfohlen. ${ }^{21}$ Zwei Jahre später, 2004, kam es zur Ausfolgung der beiden Bilder, 57 Jahre nach Beginn der erfolglosen Restitutionsbemühungen der in das US-amerikanische Exil vertriebenen Familie Kraus.

\section{Literaturverzeichnis}

Bonyhady, Tim. Wohllebengasse. Die Geschichte meiner Wiener Familie. Wien: Zsolnay, 2013. Christie's Wien (Hg.). Benefit Sale on Behalf of the Federation of Jewish Communities of Austria. 1996.

Lillie, Sophie. Was einmal war. Handbuch der enteigneten Kunstsammlungen Wiens. Wien: Czernin, 2003. 596-600.

Lillie, Sophie. „,Herrenlos‘? Die ungeklärte Akte Mauerbach“. Recollecting. Raub und Restitution. Hg. Alexandra Reininghaus. Wien: Passagen, 2008. 211-223.

Mayer, Monika. „Bruno Grimschitz und die Österreichische Galerie 1938-1945. Eine biographische Annäherung im Kontext der aktuellen Provenienzforschung“. NS-Kunstraub in Österreich und die Folgen. Hg. Gabriele Anderl und Alexandra Caruso. Innsbruck: Studienverlag, 2005. 59-79.

Mayer, Monika. „,Wien ist um einen bedeutsamen Kunstschatz reicher geworden. ' Zur Geschichte einer Institution. Von der Modernen Galerie 1903 zur Österreichischen Galerie Belvedere“. Meisterwerke des Belvedere. Hg. Stella Rollig. Wien: Belvedere, 2018a. 36-41. Mayer, Monika. „,Treuhänderische“ Übergaben von Kunstwerken an die Österreichische Galerie im Kontext der aktuellen Provenienzforschung“. Treuhänderische Übernahme und Verwahrung. International und interdisziplinär betrachtet. Hg. Olivia Kaiser, Christina Köstner-Pemsel und Markus Stumpf. Göttingen: V\&R unipress, 2018b. 187-199.

Schulz, Bernhard. „Auf der anderen Seite der Leinwand. Vorbildlich: das ,Raubkunst‘-Projekt des Hamburger Museums für Kunst und Gewerbe“. Potsdamer Neueste Nachrichten, 21.10.2014.

Schwarz, Birgit. Hitlers Museum. Die Fotoalben Gemäldegalerie Linz: Dokumente zum „Führermuseum“. Wien, Köln, Weimar: Böhlau, 2004.

Wladika, Michael. „Die Beanspruchung von Kunst- und Kulturgegenständen durch die Sammelstellen 1959-1972“. Treuhänderische Übernahme und Verwahrung. International und interdisziplinär betrachtet. Hg. Olivia Kaiser, Christina Köstner-Pemsel und Markus Stumpf. Göttingen: V\&R unipress, 2018. 85-98.

21 Vgl. http://www.provenienzforschung.gv.at/beiratsbeschluesse/Kraus_Gottlieb_2002-06-19. pdf (25.11.2019). 


\section{Archiv-Quellen}

Archiv Belvedere, A: Archiv der Österreichischen Galerie Belvedere, Zl. 134/1942; Abschrift der Rechnung der Vugesta an die Österreichische Galerie vom 14. April 1942.

Archiv Belvedere, B: Archiv der Österreichischen Galerie Belvedere, Zl. 318/1946; Schreiben Fritz Novotny an das Magistratische Bezirksamt für den I. Bezirk vom 3. Dezember 1946.

Archiv Belvedere, C: Archiv der Österreichischen Galerie Belvedere, Zl. 142/1949; Schreiben der Direktion der Österreichischen Galerie an das Bundesdenkmalamt vom 17. März 1949.

Archiv Belvedere, D: Archiv der Österreichischen Galerie Belvedere, Zl. 702/1963; Schreiben des Bundesdenkmalamts an die Direktion der Österreichischen Galerie vom 17. Juli 1963.

Archiv Belvedere, E: Archiv der Österreichischen Galerie Belvedere, Zl. 466/1965; Schreiben des Bundesministeriums für Unterricht an die Direktion des Kunsthistorischen Museums vom 25. März 1965.

Archiv BDA, A: Archiv des Bundesdenkmalamtes, Wohnungsanforderungsakten; Gottlieb Kraus, Zl. 66/1923.

Archiv BDA, B: Archiv des Bundesdenkmalamtes, Restitutionsmaterialien, Karton 8/1, Kraus; Liste der aus dem Lager Kirchner übernommenen Bilder, 19. Dezember 1941.

Archiv BDA, C: Archiv des Bundesdenkmalamtes, Restitutionsmaterialien, Karton 10, Mappe 10; Schreiben Herbert Seiberl an Hans Posse vom 7. Jänner 1942.

Archiv BDA, D: Archiv des Bundesdenkmalamtes, Restitutionsmaterialien, Karton 10, Mappe 10; Schreiben Hans Posse an Herbert Seiberl vom 4. März 1942.

Archiv BDA, E: Archiv des Bundesdenkmalamtes, Restitutionsmaterialien, Karton 12/2, Mappe 5a; undatiertes „Verzeichnis der mit dem 16. und 17. amerikanischen Transport vom 15.III.1948 von München nach Salzburg rückgeführten Kunstgegenstände (VugestaErwerbungen)“.

Bundesarchiv, A: Bundesarchiv Koblenz B 323, Zl. 142; Schreiben Karl Herber an Hans Posse vom 5. März 1942.

Bundesarchiv, B: Bundesarchiv Koblenz, B 323, Zl. 474; Schreiben der Treuhandverwaltung von Kulturgut München an Rechtsanwalt Hanns Hügel vom 18. September 1961.

Staatsarchiv, A: Österreichisches Staatsarchiv, Archiv der Republik, Bundesministerium für Finanzen, Akten der Vermögensverkehrsstelle, Zl. 41.581 u. 41.582.

Staatsarchiv, B: Österreichisches Staatsarchiv, Archiv der Republik, Bundesministerium für Finanzen, Akten der Vermögensverkehrsstelle, Zl. 41.582; Beschlagnahmeverfügung der Gestapo vom 13. Juni 1941, Zl. 3.003/1941.

Staatsarchiv, C: Österreichisches Staatsarchiv, Archiv der Republik, Bundesministerium für Finanzen; Journalbücher der Vugesta, Band 7.

Staatsarchiv, D: Österreichisches Staatsarchiv, Archiv der Republik, Bundesministerium für Finanzen, Akten der Finanzlandesdirektion für Wien, Niederösterreich und Burgenland, Zl. 19.633; Vollmacht von Gottlieb, Mathilde und Marie Kraus, Washington, vom 11. Juli 1947.

Staatsarchiv, E: Österreichisches Staatsarchiv, Archiv der Republik, Bundesministerium für Finanzen, Finanzprokuratur, Zl. 40.378; Schreiben des Bundesministeriums für Finanzen an die Finanzprokuratur vom 18. Juli 1961.

Steiermärkisches Landesarchiv, A: Steiermärkisches Landesarchiv, Neue Galerie Akten, Sch. 17; Schreiben Rechtsanwalt Otto Scheff an den Direktor der Neuen Galerie, Hans Riehl, vom 6. März 1956. 